Received: 15.12 .2020

Revised: 29.01 .2021

Accepted: 26.02 .2021

DOI: $10.17804 / 2410-9908.2021 .1 .006-025$

\title{
A CLASS OF EXACT SOLUTIONS WITH SPATIAL ACCELERATION FOR THE DESCRIPTION OF VISCOUS INCOMPRESSIBLE FLUID FLOWS IN THE FIELD OF MASS FORCES
}

\author{
N. V. Burmasheva ${ }^{\text {a** }}$, E. Yu. Prosviryakov ${ }^{\text {b) }}$ \\ Institute of Engineering Science, Ural Branch of the Russian Academy of Sciences, \\ 34 Komsomolskaya St., Ekaterinburg, 620049, Russian Federation \\ a) (iD https://orcid.org/0000-0003-4711-1894 nat_burm@mail.ru; \\ b) iD https://orcid.org/0000-0002-2349-7801 ख evgen_pros@mail.ru \\ *Corresponding author. E-mail: nat_burm@mail.ru \\ Address for correspondence: 34 Komsomolskaya St., Ekaterinburg, 620049, Russian Federation \\ Tel.: +7 (343) 37535 76; fax: +7 (343) 3745330
}

The article presents a new class of exact solutions to the system of Navier-Stokes equations, which allows one to take into account the nonlinear distribution of the pressure field and the influence of external volumetric forces, as well as the possibility of horizontal fluid outflow/inflow when modeling its vertical motion. This class is a generalization of the Lin-Sidorov-Aristov class, which assumes the linear distribution of two projections of the fluid flow velocity vector along a part of the coordinates and the independence of the third projection of the velocity vector from these coordinates.

Keywords: class of exact solutions, Navier-Stokes equation, overdetermined system, field of mass (volumetric) forces.

\section{References}

1. Shablovsky O.N. Spherical flow of an ideal fluid in a spatially nonuniform field of force. Vestnik Tomskogo Gosudarstvennogo Universiteta. Matematika i Mekhanika [Tomsk State University Journal of Mathematics and Mechanics], 2020, vol. 64. pp. 146-155. DOI 10.17223/19988621/64/11. (In Russian).

2. Manuylovich S.V. Longitudinal-periodic flow of a viscous fluid generated by a wall volumetric force. Izvestiya RAN. Mekhanika Zhidkosti i Gaza, 2015, no. 4, pp. 59-67. (In Russian).

3. Manuylovich S.V. Cross-flow control in the boundary layer on a sliding wing using a wall volumetric force. Izvestiya RAN. Mekhanika Zhidkosti i Gaza, 2020, no. 1, pp. 45-56. DOI: 10.31857/S0568528120010090. (In Russian).

4. $\quad$ Rosensweig R.E. Magnetic fluids. Ann. Rev. Fluid Mech., 1987, vol. 19, no. 1, pp. 437-463. DOI: 10.1146/annurev.fl.19.010187.002253.

5. Rosensweig R.E. Ferrohydrodynamics, Cambridge, Cambridge University Press, 1985, 344 p.

6. Yerin C.V., Vivchar V.I. Ellipsometry of magnetic fluid in a magnetic field. Journal of Magnetism and Magnetic Materials, 2020, vol. 498, pp. 166144. DOI: 10.1016/j.jmmm.2019.166144.

7. Wei Pan, Jianghong Gong. Effects of magnetic fluid on magnetic fluid damper. Key Engineering Materials, 2012, vols. 512-515, pp. 1479-1483. DOI: 10.4028/www.scientific.net/KEM.512515.1479 .

8. Safarik I., Prochazkova J., Baldikova E., Timko M., Kopcansky P., Rajnak M., Torma N., Pospiskova K. Modification of diamagneticmaterials using magnetic fluids. Ukr. J. Phys., 2020, vol. 65, no. 9, pp. 751-760. DOI: 10.15407/ujpe65.9.751. 
9. Horváth B., Szalai I. Nonlinear magnetic properties of magnetic fluids for automotive applications. Hungarian Journal of Industry and Chemistry, 2020, vol. 48, no. 1, pp. 61-65. DOI: $10.33927 /$ hjic-2020-09.

10. Bratsun D.A., Vyatkin V.A., Mukhamatullin A.R. On exact nonstationary solutions of equations of vibrational convection. Computational Continuum Mechanics, 2017, vol. 10, no. 4, pp. 433-444. DOI: 10.7242/1999-6691/2017.10.4.35. (In Russian).

11. Polezhaev V.I., Bello M.S., Verezub N.A., Dubovik K.G., Lebedev A.P., Nikitin S.A., Pavlovskiy D.S., Fedyushkin A.I. Konvektivnye protsessy v nevesomosti [Convective Processes under Microgravity]. Moscow, Nauka Publ., 1991, 242 p. (In Russian).

12. Polezhaev V.I., Sazonov V.V. Mechanics of Weightlessness and Gravity Sensitive Systems. Annotatsii dokl. nauch.-issled. seminara [Abstracts of Scientific Research Workshop, Preprint no. 898]. Moscow, IPM im. M.V. Keldysha Publ, 2009, 36 p. (In Russian).

13. Surzhikov S.T. Giperzvukovoe obtekanie razrezhennym gazom poverkhnostnogo tleyushchego razryadas vneshnim magnitnym polem [Hypersonic Rarefied Gas Flow Past a Surface Glow Discharge with an External Magnetic Field]. Moscow, Institut Problem Mekhaniki RAN Publ., 2011, 274 p. (In Russian).

14. Kedrinskiy V.K. Role of nuclei density as a "hidden" parameter in the formation of anomalous zones in a heavy cavitating magma. Journal of Applied Mechanics and Technical Physics, 2014, vol. 55, pp. 276-281. DOI: 10.1134/S0021894414020102.

15. Afonin V.V., Petrov V.Y. On exact solutions for a Luttinger liquid with one impurity. Jetp Lett., 2019, vol. 109, pp. 762-767. DOI: 10.1134/S0021364019110031.

16. Kouwn S., Moon T., Oh P. Exact solution and exotic fluid in cosmology. Entropy, 2012, vol. 14, pp. 1771-1783. DOI: 10.3390/e14091771.

17. Aristov S.N. Stationary cylindrical vortex in a viscous fluid. Dokl. Akad. Nauk, 2001, vol. 377, pp. 477-480. (In Russian).

18. Aristov S.N. Exact solution of the point source problem. Doklady Mathematics, 1995, vol. 40, no. 7, pp. 346-348. (In Russian).

19. Aristov S.N. Three-Dimensional Conical Viscous Incompressible Fluid Flows. Fluid Dynamics, 1998, vol. 33, no. 929. DOI: 10.1007/BF02698663.

20. Aristov S.N., Grabovskii V.I. Self-similar solution of the Navier-Stokes equations governing gas flows in rotary logarithmically spiral two-dimensional channels. Fluid Dyn., 1995, vol. 30, pp. 838-844. DOI: 10.1007/BF02078198.

21. Temam R. Navier-Stokes Equations and Nonlinear Functional Analysis, Society for Industrial Mathematics, 1987, $155 \mathrm{p}$.

22. Landau L.D., Lifshitz E.M. Fluid Mechanics: Vol. 6 (Course of Theoretical Physics S), 2nd Edition. Butterworth-Heinemann, 1987, 560 p.

23. Khmelnik S.I. Navier-Stokes equations. On the existence and the search method for global solutions. Israel, Mathematics in Computer Comp., 2010, 106 p. (In Russian).

24. Rouse H. Advanced mechanics of fluids, New York, Wiley, 1959, 474 p.

25. Boussinesq J. Théorie de l'écoulement tourbillonnant et tumultueux des liquides dans les lits rectilignes a grande section, Paris, Gauthier-Villars et fils, 1897, 90 p.

26. Gershuni G.Z., Zhukhovitskii E.M. Convective stability of incompressible fluids. Israel Program for Scientific Translations. Jerusalem: Keter Publishing House, 1976, 330 p.

27. Müller P. Boussinesq approximation. In: The Equations of Oceanic Motions, Cambridge, Cambridge University Press, 2006, pp. 119-126. DOI: 10.1017/CBO9780511617843.012.

28. Burmasheva N.V., Prosviryakov E.Y. Exact solution for stable convective concentration flows of a Couette type. Computational Continuum Mechanics, 2020, vol. 13, no. 3, pp. 337-349. DOI: 10.7242/1999-6691/2020.13.3.27. (In Russian). 
29. Burmasheva N.V., Prosviryakov E.Y. On Marangoni shear convective flows of inhomogeneous viscous incompressible fluids in view of the Soret effect. Journal of King Saud University Science, 2020, vol. 32, no. 8, pp. 3364-3371 DOI: 10.1016/j.jksus.2020.09.023.

30. Martyushev S.G., Sheremet M.A. Conjugate natural convection combined with surface thermal radiationin a three-dimensional enclosure with a heat source. International Journal of Heat and Mass Transfer, 2014, vol. 73, pp. 340-353. DOI: 10.1016/j.ijheatmasstransfer.2014.02.009.

31. Couette M. Etudes sur le frottement des liquids. Ann. Chim. Phys., 1890, vol. 21, pp. 433-510.

32. Poiseuille J. Recherches experimentelles sur le mouvement des liquides dans les tubes de tres petits diametres. Comptes Rendus, 1840, vol. 11, pp. 961-967.

33. Stokes G.G. On the effct of the internal friction of fluid on the motion of pendulums. Camb. Philo. Trans., 1851, vol. 9, pp. 8-106.

34. Lin C.C. Note on a class of exact solutions in magneto-hydrodynamics. Arch. Rational Mech. Anal., 1958, vol. 1, pp. 391-395.

35. Shmiglevskiy Yu.D. Analiticheskie issledovaniya dinamiki gaza i zhidkosti [Analytical Studies of Fluid and Gas Dynamics]. Moscow, Editorial URSS, 1999, 231 p.

36. Sidorov A.F. Two classes of solutions of the fluid and gas mechanics equations and their connection to traveling wave theory. J. Appl. Mech. Tech. Phys., 1989, vol. 30, no. 2, pp. 197-203. DOI: $10.1007 / \mathrm{BF} 00852164$.

37. Aristov S.N. Eddy currents in thin liquid layers. Dr. Phys. \& Math. Sci. Thesis. Vladivostok, 1990, $330 \mathrm{pp}$.

38. Aristov S.N., Prosviryakov E.Yu. A new class of exact solutions for three-dimensional thermal diffusion equations. Theoretical Foundations of Chemical Engineering, 2016, vol. 50, no. 3, pp. 286-293. DOI: 10.1134/S0040579516030027.

39. Prosviryakov E.Yu. A sufficient condition for the absence of strong and weak discontinuities in gas flows in flat channels. Diagnostics, Resource and Mechanics of materials and structures, 2019, iss. 3, pp. 25-40. DOI: 10.17804/2410-9908.2019.3.025-040.

40. Aristov S.N., Prosviryakov E.Yu. Large-scale flows of viscous incompressible vortical fluid. Russian Aeronautics, 2015, vol. 58, no. 4, pp. 413-418. DOI: 10.3103/S1068799815040091.

41. Privalova V.V., Prosviryakov E.Yu. Vortex flows of a viscous incompressible fluid at constant vertical velocity under perfect slip conditions. Diagnostics, Resource and Mechanics of materials and structures, 2019, iss. 2, pp. 57-70. DOI: 10.17804/2410-9908.2019.2.057-070.

42. Privalova V.V., Prosviryakov E.Yu. Exact solutions for the convective creep CouetteHiemenz flow with the linear temperature distribution on the upper border. Diagnostics, Resource and Mechanics of materials and structures, 2018, iss. 2, pp. 92-109. DOI: 10.17804/24109908.2018.2.092-109.

43. Privalova V.V. and Prosviryakov E.Yu. The effect of tangential boundary stresses on the convective unidirectional flow of a viscous fluid layer under the lower boundary heating condition. Diagnostics, Resource and Mechanics of materials and structures, 2019, iss. 4, pp. 44-55. DOI: 10.17804/2410-9908.2019.4.044-055.

44. Burmasheva N.V., Prosviryakov E.Yu. An exact solution to the description of a unidirectional Marangoni flow of a viscous incompressible fluid with the Navier boundary condition. Velocity field investigation. Diagnostics, Resource and Mechanics of materials and structures, 2019, iss. 5, pp. 23-39. DOI: 10.17804/2410-9908.2019.5.023-039.

45. Burmasheva N.V., Prosviryakov E.Yu. An exact solution for describing the unidirectional Marangoni flow of a viscous incompressible fluid with the Navier boundary condition. Temperature field investigation. Diagnostics, Resource and Mechanics of materials and structures, 2020, iss. 1, pp. 6-23. DOI: 10.17804/2410-9908.2020.1.006-023.

46. Burmasheva N.V., Prosviryakov E.Yu. Exact solution for describing a unidirectional Marangoni flow of a viscous incompressible fluid with the Navier boundary condition. Pressure field 
investigation. Diagnostics, Resource and Mechanics of materials and structures, 2020, iss. 2, pp. 61-75. DOI: 10.17804/2410-9908.2020.2.061-075.

47. Burmasheva N.V., Prosviryakov E.Yu. Temperature field investigation in layered flows of a vertically swirling viscous incompressible fluid under two thermocapillar forces at a free boundary. Diagnostics, Resource and Mechanics of materials and structures, 2019, iss. 1, pp. 6-42. DOI: 10.17804/2410-9908.2019.1.006-042.

48. Gorshkov A.V., Prosviryakov E.Y. Complex stationary convection with third-kind boundary conditions at the boundaries of a fluid layer. Diagnostics, Resource and Mechanics of materials and structures, 2016, iss. 2, pp. 34-47. DOI: 10.17804/2410-9908.2016.2.034-047.

49. Burmasheva N.V., Prosviryakov E.Yu. Exact solutions for natural convection of layered flows of a viscous incompressible fluid with Specified tangential forces and the linear distribution of temperature on the layer boundaries. Diagnostics, Resource and Mechanics of materials and structures, 2017, iss. 4, pp. 16-31. DOI: 10.17804/2410-9908.2017.4.016-031.

50. Petrov A.G. Exact solution of the Navier-Stokes equations in a fluid layer between the moving parallel plates. Journal of Applied Mechanics and Technical Physics, 2012, vol. 53, no. 5, pp. 642-646. DOI: 10.1134/S0021894412050021.

51. Zyryanov V.N. Teoriya ustanovivshikhsya okeanicheskikh techeniy [Theory of steady ocean currents]. Leningrad, Gidrometeoizdat Publ., 1985. (In Russian).

52. Burmasheva N.V., Prosviryakov E.Yu. Exact solution of Navier-Stokes equations describing spatially inhomogeneous flows of a rotating fluid. Trudy Instituta Matematiki i Mekhaniki UrO RAN, 2020, vol. 26, no. 2, pp. 79-87. DOI: 10.21538/0134-4889-2020-26-2-79-87. (In Russian).

53. Burmasheva N.V., Prosviryakov E.Yu. A class of exact solutions for two-dimensional equations of geophysical hydrodynamics with two Coriolis parameters. Izvestiya Irkutskogo gosudarstvennogo universiteta. Seriya "Matematika" [The Bulletin of Irkutsk State University. Series "Mathematics"], 2020, vol. 32, pp. 33-48. DOI: 10.26516/1997-7670.2020.32.33. (In Russian).

54. Burmasheva N.V., Prosviryakov E.Yu. Isothermal layered flows of a viscous incompressible fluid with spatial acceleration in the case of three Coriolis parameters. Diagnostics, Resource and Mechanics of materials and structures, 2020, iss. 3, pp. 29-46. DOI: 10.17804/24109908.2020.3.029-046.

55. Meleshko S.V., Pukhnachev V.V. On a class of partially invariant solutions of the NavierStokes equations. Journal of Applied Mechanics and Technical Physics, 1999, vol. 40, no. 2, pp. 208-216.

56. Pukhnachev V.V. Symmetries in the Navier-Stokes equations. Uspekhi Mekhaniki, 2006, vol. 1, pp. 3-76. (In Russian).

57. Aristov S.N., Gitman I.M. Viscous flow between two moving parallel disk: exact solutions and stability analysis. J. Fluid Mech., 2002, vol. 464, p. 209-215. DOI: 10.1017/S0022112002001003.

58. Berker R. An exact solution of the Navier-Stokes equation - the vortex with curvilinear axis. Int. J. Eng. Sci. 1982, vol. 20, p. 217-230.

59. Berker R. A new solution of the Navier-Stokes equation for the motion of a fluid contained between two parallel plates rotating about the same axis. Arch. Mech., 1979, vol. 31, pp. 265-280.

60. Dorrepaal J.M. An exact solution of the Navier-Stokes equation which describes nonorthogonal stagnation point flow in two dimensions. J. Fluid Mech., 1986, vol. 163, p. 141-147.

61. Ekman V.W. On the influence of the earth's rotation on ocean currents. Ark. Mat. Astron. Fys., 1905, vol. 2, pp. 1-52.

62. Gupta A.S. Ekman layer on a porous plate. Phys. F1uids, 1972, vol. 15, pp. 930-931.

63. Marques F., Sanchez J., Weidman P.D. Generalized Couette-Poiseuille flow with boundary mass transfer. J. Fluid Mech., 1998, vol. 374, pp. 221-249.

64. Rajagopal K.R. A class of exact solutions to the Navier-Stokes equations. Int. J. Eng. Sci., 1984, vol. 22, pp. 451-458. 
65. Rasmussen H. Steady flow between two porous disks. ZAMP, 1970, vol. 21, pp. 187-195. 66. Wang C.Y. Exact solution of the Navier-Stokes equations-the generalized Beltrami flows, review and extension. Acta Mech., 1990, vol. 81, p. 69-74.

67. Wang C.Y. Exact solutions of the steady-state Navier-Stokes equations. Annu. Rev. Fluid Mech., 1991, vol. 23, pp. 159-177.

68. Wang C.Y. Stagnation flow with slip: Exact solution of the Navier-Stokes equations. ZAMP, 2003, vol. 54, pp. 184-189.

69. Wang, C.Y. On a class of exact solutions of the Navier-Stokes equations. J. Appl. Mech., 1966, vol. 33, pp. 696-698. 
Подана в журнал: 15.12 .2020

УДК 517.958

DOI: $10.17804 / 2410-9908.2021 .1 .006-025$

\title{
КЛАСС ТОЧНЫХ РЕШЕНИЙ С ПРОСТРАНСТВЕННЫМ УСКОРЕНИЕМ ДЛЯ ОПИСАНИЯ ТЕЧЕНИЙ ВЯЗКОЙ НЕСЖИМАЕМОЙ ЖИДКОСТИ В ПОЛЕ МАССОВЫХ СИЛ
}

\author{
Н. В. Бурмашева ${ }^{\text {a)* }}$ Е. Ю. Просвиряков ${ }^{\text {) }}$ \\ Институт машиноведения УрО РАН, \\ 34, ул. Комсомольская, г. Екатеринбург, Российская Федерация \\ a) (iD https://orcid.org/0000-0003-4711-1894 nat_burm@mail.ru; \\ б) (iD https://orcid.org/0000-0002-2349-7801 ख evgen_pros@mail.ru \\ *Ответственный автор. Электронная почта: nat_burm@mail.ru \\ Адрес для переписки: ул. Комсомольская, 34, Екатеринбург, Российская Федерация \\ Тел.: +7 (343) 375-35-76; факс: +7 (343) 374-53-30
}

В статье представлен новый класс точных решений системы уравнений Навье-Стокса, позволяющий учитывать нелинейное распределение поля давления и влияние внешних объемных сил, а также принимать во внимание возможность горизонтального оттока/притока жидкости при моделировании ее движения в вертикальном направлении. Данный класс является обобщением класса Линя-Сидорова-Аристова, предполагающего линейное распределение двух проекций вектора скорости течения жидкости по части координат и независимость от этих координат третьей проекции вектора скорости.

Ключевые слова: класс точных решений, уравнение Навье-Стокса, переопределенная система, поле массовых (объемных) сил.

\section{1. Введение}

Течение реальных жидкостей не изолировано от влияния окружающего мира. Любое течение жидкости подвержено влиянию незримых полей, наличие которых тем не менее регистрируется соответствующей аппаратурой. Примером такого поля является поле тяготения. Помимо силы тяжести большое влияние на характеристики течения оказывают также магнитное и электрическое поля, особенно когда речь идет о течении ферромагнитных жидкостей [1-20]. Жидкости такого типа не существуют в природе, они были созданы искусственным путем посредством добавления измельченного магнитного материала в жидкостьноситель при поиске способов перемещения ракетного топлива в условиях низкой гравитации [5]. Ввиду структуры данного типа сред (твердые частицы + жидкость-носитель) оказывается принципиально важным также учитывать и распределение поля концентрации твердых частиц, входящих в состав. Таким образом, класс силовых и других полей, индуцирующих движение жидкости, а значит, и влияющих на ее кинематические характеристики, достаточно широк. Кроме того, интенсивность указанных полей имеет существенно неоднородное и нелинейное распределение в пространстве, что также приводит к необходимости учета этих факторов в соотношениях, описывающих динамику жидкостей.

Традиционно при описании течений вязких жидкостей используется модель несжимаемой вязкой жидкости, состоящая из уравнения Навье-Стокса и уравнения несжимаемости [21-24]. Кроме того, довольно распространенным является подход, основанный на гипотезе Буссинеска [25-30], суть которого заключается в замене плотности жидкости линейной функцией приращения температуры (и/или концентрации в общем случае) в представлении для силы тяжести. При этом в случае изотермического процесса (за счет нулевого приращения температу- 
ры) получается, что член уравнения Навье-Стокса, отвечающий за влияние силы тяжести, отбрасывается. На самом деле это не так, влияние гравитации на параметры течения сохраняется. Оно пропадает только в случае движения жидкости в условиях невесомости. При отсутствии гравитации (или при микрогравитации) на первый план как раз выходят электрические и магнитные воздействия на жидкость, а также воздействия инерционной природы. Система уравнений указанной математической модели является квадратично нелинейной, что значительно затрудняет нахождение точных решений, поскольку не всегда очевидным является даже факт существования решения, не говоря уже о том, какова его структура.

Основным подходом в этом направлении считается метод построения семейств точных решений уравнений гидродинамики. Одними из первых точных решений были решения Куэтта [31], Пуазейля [32] и Стокса, описанные им в виде двух задач [33]. Эти решения являются частными случаями класса, объединяющего функции, линейные по части координат:

$$
\begin{gathered}
V_{x}(x, y, z, t)=U(z, t)+u_{1}(z, t) x+u_{2}(z, t) y ; \\
V_{y}(x, y, z, t)=V(z, t)+v_{1}(z, t) x+v_{2}(z, t) y ; \\
V_{z}=W(z, t) .
\end{gathered}
$$

Класс (1) был предложен для задач магнитной гидродинамики Линем [34]. Достоинством данного класса является относительно простая структура, если говорить о зависимости от пространственных координат $x, y$, но при этом данный класс способен описывать существенно трехмерные течения со сложной динамикой за счет зависимости общего вида от вертикальной координаты $z$ и временной координаты $t$. Однако при всех его положительных сторонах предложенный класс не учитывает влияние внешних массовых сил и наличие пространственных ускорений [35] для вертикальной скорости $V_{z}$.

Класс Линя широко распространен и при решении задач конвекции $[36,37]$ и задач термодиффузии [38]. Стоит отметить, что при рассмотрении этих проблем значительно усложняются сами уравнения определяющей системы (по сравнению с изотермическим случаем) ввиду учета дополнительных (тепловых, концентрационных) факторов, а также увеличивается число соотношений, входящих в базовую систему уравнений: добавляются уравнение теплопроводности и/или уравнение, описывающее изменение концентрации при течении жидкости. Это приводит к тому, что в случае неизотермических задач исследователи преимущественно рассматривают частные случаи класса Линя, в которых либо игнорируют вертикальную скорость [39, 40], либо полагают равными нулю некоторые (или все) пространственные ускорения [41, 42], либо принимают оба упрощения совместно [43-50].

В настоящей работе приводится класс точных решений, обобщающий семейство (1) за счет учета неоднородности распределения третьей компоненты вектора скорости и представления давления и внешнего поля объемных сил в виде полной кубики от двух пространственных координат с зависимостями общего вида их коэффициентов от времени и третьей пространственной координаты.

\section{2. Постановка задачи}

Нестационарное изотермическое течение вязкой несжимаемой жидкости, движущейся в силовом поле, описывается уравнением Навье-Стокса, дополненным уравнением несжимаемости [21-24]:

$$
\begin{gathered}
\frac{\partial \boldsymbol{V}}{\partial t}+(\boldsymbol{V}, \nabla) \boldsymbol{V}=-\nabla P+v \Delta \boldsymbol{V}+\boldsymbol{F} ; \\
(\nabla, \boldsymbol{V})=0 .
\end{gathered}
$$

Burmasheva N. V., Prosviryakov E. Yu. A class of exact solutions with spatial acceleration for the description of viscous incompressible fluid flows in the field of mass forces // Diagnostics, Resource and Mechanics of materials and structures. 2021. - Iss. 1. - P. 6-25. - DOI: 10.17804/2410-9908.2021.1.006-025. 
В уравнении Навье-Стокса (2) и уравнении несжимаемости (3) приняты обозначения: $\boldsymbol{V}=\left(V_{x}, V_{y}, V_{z}\right)$ - вектор скорости; $\boldsymbol{P}$ - нормированное на постоянную плотность жидкости отклонение давления от гидростатического значения; $v$ - кинематическая (молекулярная) вязкость жидкости; $\boldsymbol{F}=\left(F_{x}, F_{y}, F_{z}\right)$ - вектор объемной (массовой) силы; $\nabla=(\partial / \partial x, \partial / \partial y, \partial / \partial z)-$ оператор Гамильтона; $(\boldsymbol{V}, \nabla)=\left(V_{x} \frac{\partial}{\partial x}+V_{y} \frac{\partial}{\partial y}+V_{z} \frac{\partial}{\partial z}\right)-$ конвективная производная; $\Delta=\partial^{2} / \partial x^{2}+\partial^{2} / \partial y^{2}+\partial^{2} / \partial z^{2}-$ оператор Лапласа. Заметим, что при записи уравнений (2) поле массовых сил $\boldsymbol{F}$ не является потенциальным. Без ограничения общности полагаем, что оно соленоидально. Если поле является потенциальным, то его учет можно реализовать, вводя модифицированное давление [26, 51].

Координатная форма записи системы уравнений (2)-(3) имеет вид:

$$
\begin{gathered}
\frac{\partial V_{x}}{\partial t}+V_{x} \frac{\partial V_{x}}{\partial x}+V_{y} \frac{\partial V_{x}}{\partial y}+V_{z} \frac{\partial V_{x}}{\partial z}=-\frac{\partial P}{\partial x}+v\left(\frac{\partial^{2} V_{x}}{\partial x^{2}}+\frac{\partial^{2} V_{x}}{\partial y^{2}}+\frac{\partial^{2} V_{x}}{\partial z^{2}}\right)+F_{x} \\
\frac{\partial V_{y}}{\partial t}+V_{x} \frac{\partial V_{y}}{\partial x}+V_{y} \frac{\partial V_{y}}{\partial y}+V_{z} \frac{\partial V_{y}}{\partial z}=-\frac{\partial P}{\partial y}+v\left(\frac{\partial^{2} V_{y}}{\partial x^{2}}+\frac{\partial^{2} V_{y}}{\partial y^{2}}+\frac{\partial^{2} V_{y}}{\partial z^{2}}\right)+F_{y} \\
\frac{\partial V_{z}}{\partial t}+V_{x} \frac{\partial V_{z}}{\partial x}+V_{y} \frac{\partial V_{z}}{\partial y}+V_{z} \frac{\partial V_{z}}{\partial z}=-\frac{\partial P}{\partial z}+v\left(\frac{\partial^{2} V_{z}}{\partial x^{2}}+\frac{\partial^{2} V_{z}}{\partial y^{2}}+\frac{\partial^{2} V_{z}}{\partial z^{2}}\right)+F_{z} \\
\frac{\partial V_{x}}{\partial x}+\frac{\partial V_{y}}{\partial y}+\frac{\partial V_{z}}{\partial z}=0 .
\end{gathered}
$$

Каждое из уравнений системы (4)-(7) определяет связь между неизвестными этой системы, в роли которых выступают компоненты $V_{x}, V_{y}, V_{z}$ вектора скорости и поле давления $P$ при известном распределении массовой силы $\boldsymbol{F}$. Данные связи преимущественно имеют нелинейный характер, определяемый членами конвективной производной.

\section{3. Класс точных решений}

Течения реальных жидкостей характеризуются неоднородным распределением кинетико-силовых параметров внутри и на границах области течения. По этой причине будем искать точное решение уравнений (4)-(7) в виде следующих форм [40, 52-69]:

$$
\begin{gathered}
V_{x}=U(z, t)+u_{1}(z, t) x+u_{2}(z, t) y ; \\
V_{y}=V(z, t)+v_{1}(z, t) x+v_{2}(z, t) y ; \\
V_{z}=W(z, t)+w_{1}(z, t) x+w_{2}(z, t) y ; \\
P=P_{0}(z, t)+P_{1}(z, t) x+P_{2}(z, t) y+P_{11}(z, t) \frac{x^{2}}{2}+P_{12}(z, t) x y+P_{22}(z, t) \frac{y^{2}}{2}+ \\
+P_{111}(z, t) \frac{x^{3}}{6}+P_{112}(z, t) \frac{x^{2}}{2} y+P_{122}(z, t) x \frac{y^{2}}{2}+P_{222}(z, t) \frac{y^{3}}{6}
\end{gathered}
$$

Burmasheva N. V., Prosviryakov E. Yu. A class of exact solutions with spatial acceleration for the description of viscous incompressible fluid flows in the field of mass forces // Diagnostics, Resource and Mechanics of materials and structures. 2021. - Iss. 1. - P. 6-25. - DOI: 10.17804/2410-9908.2021.1.006-025. 


$$
\begin{gathered}
F_{x}=F_{0}^{x}(z, t)+F_{1}^{x}(z, t) x+F_{2}^{x}(z, t) y+F_{11}^{x}(z, t) x^{2}+F_{12}^{x}(z, t) x y+F_{22}^{x}(z, t) y^{2}+ \\
+F_{111}^{x}(z, t) x^{3}+F_{112}^{x}(z, t) x^{2} y+F_{122}^{x}(z, t) x y^{2}+F_{222}^{x}(z, t) y^{3} ; \\
\begin{array}{c}
F_{y}=F_{0}^{y}(z, t)+F_{1}^{y}(z, t) x+F_{2}^{y}(z, t) y+F_{11}^{y}(z, t) x^{2}+F_{12}^{y}(z, t) x y+F_{22}^{y}(z, t) y^{2}+ \\
+F_{111}^{y}(z, t) x^{3}+F_{112}^{y}(z, t) x^{2} y+F_{122}^{y}(z, t) x y^{2}+F_{222}^{y}(z, t) y^{3} ; \\
F_{z}=F_{0}^{z}(z, t)+F_{1}^{z}(z, t) x+F_{2}^{z}(z, t) y+F_{11}^{z}(z, t) x^{2}+F_{12}^{z}(z, t) x y+F_{22}^{z}(z, t) y^{2}+ \\
+F_{111}^{z}(z, t) x^{3}+F_{112}^{z}(z, t) x^{2} y+F_{122}^{z}(z, t) x y^{2}+F_{222}^{z}(z, t) y^{3} .
\end{array}
\end{gathered}
$$

Поскольку коэффициенты распределений (8) зависят только от двух переменных времени $t$ и вертикальной координаты $z$, условимся всюду далее обозначать производную по времени точкой, а производную по пространственной координате $z$ - штрихом.

Вначале осуществим подстановку выражений (8) в уравнение несжимаемости (7):

$$
\begin{gathered}
\frac{\partial\left(U+u_{1} x+u_{2} y\right)}{\partial x}+\frac{\partial\left(V+v_{1} x+v_{2} y\right)}{\partial y}+\frac{\partial\left(W+w_{1} x+w_{2} y\right)}{\partial z}=0 ; \\
u_{1}+v_{2}+W^{\prime}+w_{1}^{\prime} x+w_{2}^{\prime} y=0 .
\end{gathered}
$$

С учетом независимости координат выбранной декартовой системы с необходимостью следует вывод о том, что пространственные ускорения $w_{1}, w_{2}$ могут зависеть только от времени $t$, при этом однородная составляющая скорости должна удовлетворять следующему дифференциальному уравнению:

$$
W^{\prime}=-\left(u_{1}+v_{2}\right)
$$

На основании полученных выводов можем внести корректировку в структуру рассматриваемого класса (8):

$$
\begin{gathered}
V_{x}=U(z, t)+u_{1}(z, t) x+u_{2}(z, t) y ; \\
V_{y}=V(z, t)+v_{1}(z, t) x+v_{2}(z, t) y ; \\
V_{z}=W(z, t)+w_{1}(t) x+w_{2}(t) y \\
P=P_{0}(z, t)+P_{1}(z, t) x+P_{2}(z, t) y+P_{11}(z, t) \frac{x^{2}}{2}+P_{12}(z, t) x y+P_{22}(z, t) \frac{y^{2}}{2}+ \\
+P_{111}(z, t) \frac{x^{3}}{6}+P_{112}(z, t) \frac{x^{2}}{2} y+P_{122}(z, t) x \frac{y^{2}}{2}+P_{222}(z, t) \frac{y^{3}}{6} \\
F_{x}=F_{0}^{x}(z, t)+F_{1}^{x}(z, t) x+F_{2}^{x}(z, t) y+F_{11}^{x}(z, t) x^{2}+F_{12}^{x}(z, t) x y+F_{22}^{x}(z, t) y^{2}+ \\
+F_{111}^{x}(z, t) x^{3}+F_{112}^{x}(z, t) x^{2} y+F_{122}^{x}(z, t) x y^{2}+F_{222}^{x}(z, t) y^{3} \\
F_{y}=F_{0}^{y}(z, t)+F_{1}^{y}(z, t) x+F_{2}^{y}(z, t) y+F_{11}^{y}(z, t) x^{2}+F_{12}^{y}(z, t) x y+F_{22}^{y}(z, t) y^{2}+ \\
+F_{111}^{y}(z, t) x^{3}+F_{112}^{y}(z, t) x^{2} y+F_{122}^{y}(z, t) x y^{2}+F_{222}^{y}(z, t) y^{3} \\
F_{z}=F_{0}^{z}(z, t)+F_{1}^{z}(z, t) x+F_{2}^{z}(z, t) y+F_{11}^{z}(z, t) x^{2}+F_{12}^{z}(z, t) x y+F_{22}^{z}(z, t) y^{2}+
\end{gathered}
$$




$$
+F_{111}^{z}(z, t) x^{3}+F_{112}^{z}(z, t) x^{2} y+F_{122}^{z}(z, t) x y^{2}+F_{222}^{z}(z, t) y^{3} .
$$

Подставим далее уточненный класс (10) в первое уравнение Навье-Стокса (4) и вычислим соответствующие производные:

$$
\begin{gathered}
\dot{U}+\dot{u}_{1} x+\dot{u}_{2} y+\left(U+u_{1} x+u_{2} y\right) u_{1}+\left(V+v_{1} x+v_{2} y\right) u_{2}+\left(W+w_{1} x+w_{2} y\right)\left(U^{\prime}+u_{1}^{\prime} x+u_{2}^{\prime} y\right)= \\
=-\left(P_{1}+P_{11} x+P_{12} y+P_{111} \frac{x^{2}}{2}+P_{112} x y+P_{122} \frac{y^{2}}{2}\right)+v\left(U^{\prime \prime}+u_{1}^{\prime \prime} x+u_{2}^{\prime \prime} y\right)+ \\
+F_{0}^{x}+F_{1}^{x} x+F_{2}^{x} y+F_{11}^{x} x^{2}+F_{12}^{x} x y+F_{22}^{x} y^{2}+F_{111}^{x} x^{3}+F_{112}^{x} x^{2} y+F_{122}^{x} x y^{2}+F_{222}^{x} y^{3} .
\end{gathered}
$$

Учитывая независимость пространственных переменных $x, y, z$, данное уравнение эквивалентно системе дифференциальных уравнений в частных производных:

$$
\begin{gathered}
\dot{U}+U u_{1}+V u_{2}+W U^{\prime}=-P_{1}+v U^{\prime \prime}+F_{0}^{x} ; \\
\dot{u}_{1}+u_{1}^{2}+u_{2} v_{1}+W u_{1}^{\prime}+U^{\prime} w_{1}=-P_{11}+v u_{1}^{\prime \prime}+F_{1}^{x} ; \\
\dot{u}_{2}+u_{1} u_{2}+u_{2} v_{2}+W u_{2}^{\prime}+U^{\prime} w_{2}=-P_{12}+v u_{2}^{\prime \prime}+F_{2}^{x} ; \\
w_{1} u_{1}^{\prime}=-\frac{P_{111}}{2}+F_{11}^{x} ; \\
w_{1} u_{2}^{\prime}+w_{2} u_{1}^{\prime}=-P_{112}+F_{12}^{x} ; \\
w_{2} u_{2}^{\prime}=-\frac{P_{122}}{2}+F_{22}^{x}, F_{111}^{x}=F_{112}^{x}=F_{122}^{x}=F_{222}^{x}=0 .
\end{gathered}
$$

Аналогичным образом подстановка класса (10) во второе уравнение Навье-Стокса (5) приводит к следующей системе уравнений:

$$
\begin{gathered}
\dot{V}+U v_{1}+V v_{2}+W V^{\prime}=-P_{2}+v V^{\prime \prime}+F_{0}^{y} ; \\
\dot{v}_{1}+u_{1} v_{1}+v_{1} v_{2}+W v_{1}^{\prime}+V^{\prime} w_{1}=-P_{12}+v v_{1}^{\prime \prime}+F_{1}^{y} ; \\
\dot{v}_{2}+u_{2} v_{1}+v_{2}^{2}+W v_{2}^{\prime}+V^{\prime} w_{2}=-P_{22}+v v_{2}^{\prime \prime}+F_{2}^{y} ; \\
w_{1} v_{1}^{\prime}=-\frac{P_{112}}{2}+F_{11}^{y} ; \\
w_{1} v_{2}^{\prime}+w_{2} v_{1}^{\prime}=-P_{122}+F_{12}^{y} ; \\
w_{2} v_{2}^{\prime}=-\frac{P_{222}}{2}+F_{22}^{y} ; \\
F_{111}^{y}=F_{112}^{y}=F_{122}^{y}=F_{222}^{y}=0 .
\end{gathered}
$$

В результате подстановки класса точных решений (10) в последнее уравнение НавьеСтокса получим систему вида:

$$
\begin{gathered}
\dot{W}+U w_{1}+V w_{2}+W W^{\prime}=-P_{0}^{\prime}+v W^{\prime \prime}+F_{0}^{z} ; \\
\dot{w}_{1}+u_{1} w_{1}+v_{1} w_{2}+w_{1} W^{\prime}=-P_{1}^{\prime}+F_{1}^{z} ; \\
\dot{w}_{2}+u_{2} w_{1}+v_{2} w_{2}+w_{2} W^{\prime}=-P_{2}^{\prime}+F_{2}^{z} ; \\
P_{11}^{\prime}=2 F_{11}^{z}, P_{12}^{\prime}=F_{12}^{z}, P_{22}^{\prime}=2 F_{22}^{z} ;
\end{gathered}
$$




$$
P_{111}{ }^{\prime}=6 F_{111}^{z}, P_{112}{ }^{\prime}=2 F_{112}^{z}, P_{122}{ }^{\prime}=2 F_{122}^{z}, P_{222}{ }^{\prime}=6 F_{222}^{z} .
$$

Таким образом, система уравнений (4)-(7) сводится к системе двадцати трех уравнений (9), (11), (12), (13) для определения девятнадцати компонент форм (10):

$$
\begin{gathered}
\dot{U}+U u_{1}+V u_{2}+W U^{\prime}=-P_{1}+v U^{\prime \prime}+F_{0}^{x} ; \\
\dot{u}_{1}+u_{1}^{2}+u_{2} v_{1}+W u_{1}^{\prime}+U^{\prime} w_{1}=-P_{11}+v u_{1}^{\prime \prime}+F_{1}^{x} ; \\
\dot{u}_{2}+u_{1} u_{2}+u_{2} v_{2}+W u_{2}^{\prime}+U^{\prime} w_{2}=-P_{12}+v u_{2}^{\prime \prime}+F_{2}^{x} ; \\
w_{1} u_{1}^{\prime}=-\frac{P_{111}}{2}+F_{11}^{x} ; \\
w_{1} u_{2}^{\prime}+w_{2} u_{1}^{\prime}=-P_{112}+F_{12}^{x} ; \\
w_{2} u_{2}^{\prime}=-\frac{P_{122}}{2}+F_{22}^{x} ; \\
\dot{v}_{1}+u_{1} v_{1}+v_{1} v_{2}+W v_{1}^{\prime}+V^{\prime} w_{1}=-P_{12}+v v_{1}^{\prime \prime}+F_{1}^{y} ; \\
\dot{v}_{2}+u_{2} v_{1}+v_{2}{ }^{2}+W v_{2}^{\prime}+V^{\prime} w_{2}=-P_{22}+v v_{2}^{\prime \prime}+F_{2}^{y} ; \\
w_{1} v_{1}^{\prime}=-\frac{P_{112}}{2}+F_{11}^{y} ; \\
w_{1} v_{2}^{\prime}+w_{2} v_{1}^{\prime}=-P_{122}+F_{12}^{y} ; \\
w_{2} v_{2}^{\prime}=-\frac{P_{222}}{2}+F_{22}^{y}, \\
P_{111}{ }^{\prime}=6 F_{111}^{z}, P_{112}{ }^{\prime}=2 F_{112}^{z}, P_{122}{ }^{\prime}=2 F_{122}^{z}, P_{222}{ }^{\prime}=6 F_{222}^{z} ; \\
W^{\prime}=-\left(u_{1}+v_{2}\right) \\
P_{1}^{\prime}=2 F_{11}^{z}, P_{12}^{\prime}=F_{1}^{z}, P_{22}^{\prime}=2 F_{22}^{z} ; \\
\dot{w}_{1}+u_{1} w_{1}+v_{1}+W W^{\prime}=-P_{0}^{\prime}+v W^{\prime \prime}+F_{1}^{z} ; \\
\dot{w}_{2}+u_{2} w_{1}+v_{2} w_{2}+w_{2} W^{\prime}=-P_{1}^{\prime}+F_{1}^{z} ;
\end{gathered}
$$

Напомним, что кубические слагаемые в разложениях $F_{x}$ и $F_{y}$ полагаются тождественно равными нулю, так как из уравнений редуцированных систем (11) и (12) непосредственно следует равенство нулю коэффициентов $F_{111}^{x}, F_{112}^{x}, F_{122}^{x}, F_{222}^{x}$ и $F_{111}^{y}, F_{112}^{y}, F_{122}^{y}, F_{222}^{y}$.

Также подчеркнем, что последние уравнения подсистемы (13) требуют согласованности вида коэффициентов при нелинейных членах поля давления и структуры коэффициентов, определяющих вектор объемных сил $\boldsymbol{F}$, по вертикальной координате $z$, но допускают зависимости произвольного вида по временной переменной $t$.

Важным частным случаем течений вязкой несжимаемой жидкости являются установившиеся течения. Такие течения характеризуются тем, что гидродинамические поля не зависят от времени $t$. Это приводит к тому, что в уравнениях переопределенной системы (14) пропадают члены, содержащие частные производные по времени:

$$
\begin{gathered}
W^{\prime}=-\left(u_{1}+v_{2}\right) ; \\
U u_{1}+V u_{2}+W U^{\prime}=-P_{1}+v U^{\prime \prime}+F_{0}^{x}
\end{gathered}
$$

Burmasheva N. V., Prosviryakov E. Yu. A class of exact solutions with spatial acceleration for the description of viscous incompressible fluid flows in the field of mass forces // Diagnostics, Resource and Mechanics of materials and structures. 2021. - Iss. 1. - P. 6-25. - DOI: 10.17804/2410-9908.2021.1.006-025. 


$$
\begin{gathered}
u_{1}^{2}+u_{2} v_{1}+W u_{1}^{\prime}+U^{\prime} w_{1}=-P_{11}+v u_{1}^{\prime \prime}+F_{1}^{x} ; \\
u_{1} u_{2}+u_{2} v_{2}+W u_{2}^{\prime}+U^{\prime} w_{2}=-P_{12}+v u_{2}^{\prime \prime}+F_{2}^{x} ; \\
w_{1} u_{1}^{\prime}=-\frac{P_{111}}{2}+F_{11}^{x} ; \\
w_{1} u_{2}^{\prime}+w_{2} u_{1}^{\prime}=-P_{112}+F_{12}^{x} ; \\
w_{2} u_{2}^{\prime}=-\frac{P_{122}}{2}+F_{22}^{x} ; \\
U v_{1}+V v_{2}+W V^{\prime}=-P_{2}+v V^{\prime \prime}+F_{0}^{y} ; \\
u_{1} v_{1}+v_{1} v_{2}+W v_{1}^{\prime}+V^{\prime} w_{1}=-P_{12}+v v_{1}^{\prime \prime}+F_{1}^{y} ; \\
u_{2} v_{1}+v_{2}{ }^{2}+W v_{2}^{\prime}+V^{\prime} w_{2}=-P_{22}+v v_{2}^{\prime \prime}+F_{2}^{y} ; \\
w_{1} v_{1}^{\prime}=-\frac{P_{112}}{2}+F_{11}^{y} ; \\
w_{1} v_{2}^{\prime}+w_{2} v_{1}^{\prime}=-P_{122}+F_{12}^{y} ; \\
w_{2} v_{2}^{\prime}=-\frac{P_{222}}{2}+F_{22}^{y} ; \\
P_{111}{ }^{\prime}=6 F_{111}^{z}, P_{112}{ }^{\prime}=2 F_{112}^{z}, P_{122}{ }^{\prime}=2 F_{122}^{z}, P_{222}{ }^{\prime}=6 F_{222}^{z} \\
P_{11}^{\prime}=2 F_{11}^{z}, P_{12}^{\prime}=F_{12}^{z}, P_{22}{ }^{\prime}=2 F_{22}^{z} ; \\
u_{1} w_{1}+v_{1}+W W_{2}+w_{1}^{\prime}=-P_{0}^{\prime}+v W^{\prime \prime}+F_{0}^{z} ; \\
u_{2} w_{1}+v_{2} w_{2}+w_{2} W^{\prime}=-P_{1}^{\prime}+F_{1}^{z} ;
\end{gathered}
$$

При этом свойство переопределенности наследуется от системы (14). Этот факт объясняется тем, что рассматриваемое свойство стационарности потока не меняет ни число неизвестных функций, ни число уравнений, а лишь немного упрощает вид последних.

\section{4. Условие разрешимости}

В системе дифференциальных уравнений (14) число неизвестных значительно меньше числа соотношений для их вычисления. Таким образом, имеет место переопределенность системы определяющих соотношений (15). В этом случае залогом успешного интегрирования рассматриваемой системы (нахождения нетривиального ее решения) является подбор условий совместности, удовлетворение которым гарантировало бы попарную непротиворечивость уравнений системы.

Один из очевидных вариантов таких условий (необязательно единственных) связан с тем фактом, что в системе (14) (или его модификации (15)) слагаемое $P_{112}$ давления $P$ определяется из двух уравнений:

$$
w_{1} u_{2}^{\prime}+w_{2} u_{1}^{\prime}=-P_{112}+F_{12}^{x}, w_{1} v_{1}^{\prime}=-\frac{P_{112}}{2}+F_{11}^{y}
$$


Таким образом, должно быть справедливо равенство

$$
w_{1} u_{2}^{\prime}+w_{2} u_{1}^{\prime}-F_{12}^{x}=2 w_{1} v_{1}^{\prime}-2 F_{11}^{y}
$$

После однократного интегрирования данного соотношения по пространственной переменной $z$ получим связь между пространственными ускорениями:

$$
w_{1} u_{2}+w_{2} u_{1}-2 w_{1} v_{1}=\int\left(F_{12}^{x}-2 F_{11}^{y}\right) d z+\alpha(t) .
$$

Аналогичным образом после изучения структуры уравнений

$$
w_{2} u_{2}^{\prime}=-\frac{P_{122}}{2}+F_{22}^{x}, w_{1} v_{2}^{\prime}+w_{2} v_{1}^{\prime}=-P_{122}+F_{12}^{y},
$$

определяющих слагаемое $P_{122}$, получим еще одно ограничение на выбор вида пространственных ускорений:

$$
w_{1} v_{2}+w_{2} v_{1}-2 w_{2} u_{2}=\int\left(F_{12}^{y}-2 F_{22}^{x}\right) d z+\beta(t) .
$$

Здесь $\alpha(t)$ и $\beta(t)$ - произвольные функции времени $t$.

\section{5. Течение Стокса}

Рассмотрим ползущее течение вязкой несжимаемой жидкости в приближении Стокса [33]. Пренебрегая в уравнении (1) конвективной производной $(\boldsymbol{V}, \boldsymbol{\nabla}) \boldsymbol{V}$, получим линейную версию уравнений Навье-Стокса - систему Стокса [26]:

$$
\frac{\partial \boldsymbol{V}}{\partial t}=-\nabla P+v \Delta \boldsymbol{V}+\boldsymbol{F}
$$

В координатной форме это уравнение принимает следующий вид:

$$
\begin{aligned}
& \frac{\partial V_{x}}{\partial t}=-\frac{\partial P}{\partial x}+v\left(\frac{\partial^{2} V_{x}}{\partial x^{2}}+\frac{\partial^{2} V_{x}}{\partial y^{2}}+\frac{\partial^{2} V_{x}}{\partial z^{2}}\right)+F_{x} \\
& \frac{\partial V_{y}}{\partial t}=-\frac{\partial P}{\partial y}+v\left(\frac{\partial^{2} V_{y}}{\partial x^{2}}+\frac{\partial^{2} V_{y}}{\partial y^{2}}+\frac{\partial^{2} V_{y}}{\partial z^{2}}\right)+F_{y} \\
& \frac{\partial V_{z}}{\partial t}=-\frac{\partial P}{\partial z}+v\left(\frac{\partial^{2} V_{z}}{\partial x^{2}}+\frac{\partial^{2} V_{z}}{\partial y^{2}}+\frac{\partial^{2} V_{z}}{\partial z^{2}}\right)+F_{z} .
\end{aligned}
$$

Уравнение несжимаемости (7) остается неизменным.

Подстановка класса (10) в систему (16) приводит к системе:

$$
\begin{gathered}
W^{\prime}=-\left(u_{1}+v_{2}\right) ; \\
\dot{U}=-P_{1}+v U^{\prime \prime}+F_{0}^{x} ; \\
\dot{u}_{1}=-P_{11}+v u_{1}^{\prime \prime}+F_{1}^{x} ;
\end{gathered}
$$




$$
\begin{gathered}
\dot{u}_{2}=-P_{12}+v u_{2}^{\prime \prime}+F_{2}^{x} ; \\
\dot{V}=-P_{2}+v V^{\prime \prime}+F_{0}^{y} ; \\
\dot{v}_{1}=-P_{12}+v v_{1}^{\prime \prime}+F_{1}^{y} ; \\
\dot{v}_{2}=-P_{22}+v v_{2}^{\prime \prime}+F_{2}^{y} ; \\
\dot{W}=-P_{0}^{\prime}+v W^{\prime \prime}+F_{0}^{z} ; \\
\dot{w}_{1}=-P_{1}^{\prime}+F_{1}^{z} ; \\
\dot{w}_{2}=-P_{2}^{\prime}+F_{2}^{z} ; \\
P_{11}^{\prime}=2 F_{11}^{z}, P_{12}{ }^{\prime}=F_{12}^{z}, P_{22}{ }^{\prime}=2 F_{22}^{z} ; \\
P_{111}=2 F_{11}^{x}, P_{112}=F_{12}^{x}, P_{122}=2 F_{22}^{x} ; \\
P_{112}=2 F_{11}^{y}, P_{122}=F_{12}^{y}, P_{222}=2 F_{22}^{y} ; \\
P_{111}{ }^{\prime}=6 F_{111}^{z} ; P_{112}{ }^{\prime}=2 F_{112}^{z} ; P_{122}{ }^{\prime}=2 F_{122}^{z} ; P_{222}{ }^{\prime}=6 F_{222}^{z} .
\end{gathered}
$$

Отметим, что уравнения подсистемы (18) позволяют определить вид (в квадратурах) нелинейных компонент поля давления по известному распределению поля массовых сил, а также получить дополнительные ограничения на коэффициенты кубических слагаемых, определяющих структуру проекций вектора объемных сил $\boldsymbol{F}$ :

$$
\begin{gathered}
P_{11}=2 \int F_{11}^{z} d z ; \\
P_{12}=\int F_{12}^{z} d z ; \\
P_{22}=2 \int F_{22}^{z} d z ; \\
P_{111}=2 F_{11}^{x}=6 \int F_{111}^{z} d z ; \\
P_{112}=F_{12}^{x}=2 F_{11}^{y}=2 \int F_{112}^{z} d z ; \\
P_{122}=2 F_{22}^{x}=F_{12}^{y}=2 \int F_{122}^{z} d z ; \\
P_{222}=2 F_{22}^{y}=6 \int F_{222}^{z} d z ;
\end{gathered}
$$

\section{6. Плоское течение Стокса}

Рассмотрим в качестве примера частный случай, полагая скорость $V_{y}=0$. При этом система (17) упрощается, а группа соотношений (19) дополняется рядом новых равенств:

$$
\begin{gathered}
W^{\prime}=-u_{1} ; \\
\dot{U}=-P_{1}+v U^{\prime \prime}+F_{0}^{x} ; \\
\dot{u}_{1}=-P_{11}+v u_{1}^{\prime \prime}+F_{1}^{x} ; \\
\dot{u}_{2}=-P_{12}+v u_{2}^{\prime \prime}+F_{2}^{x} ; \\
\dot{W}=-P_{0}^{\prime \prime}+v W^{\prime \prime}+F_{0}^{z} ; \\
\dot{w}_{1}=-P_{1}^{\prime \prime}+F_{1}^{z} ; \\
\dot{w}_{2}=-P_{2}^{\prime \prime}+F_{2}^{z} ;
\end{gathered}
$$

Burmasheva N. V., Prosviryakov E. Yu. A class of exact solutions with spatial acceleration for the description of viscous incompressible fluid flows in the field of mass forces // Diagnostics, Resource and Mechanics of materials and structures. 2021. - Iss. 1. - P. 6-25. - DOI: 10.17804/2410-9908.2021.1.006-025. 


$$
\begin{gathered}
P_{2}=F_{0}^{y} ; \\
P_{11}=2 \int F_{11}^{z} d z ; \\
P_{12}=F_{1}^{y}=\int F_{12}^{z} d z ; \\
P_{22}=F_{2}^{y}=2 \int F_{22}^{z} d z ; \\
P_{111}=2 F_{11}^{x}=6 \int F_{111}^{z} d z ; \\
P_{112}=F_{12}^{x}=2 F_{11}^{y}=2 \int F_{112}^{z} d z ; \\
P_{122}=2 F_{22}^{x}=F_{12}^{y}=2 \int F_{122}^{z} d z ; \\
P_{222}=2 F_{22}^{y}=6 \int F_{222}^{z} d z ;
\end{gathered}
$$

Уравнения (20)-(21) описывают установившееся плоское течение Стокса (течение Стокса вдоль плоскости $O x z)$.

Отметим, что переход к частному случаю $V_{y}=0$ привел к тому, что переопределенная система стала недоопределенной: в систему (20) входят семь уравнений для определения восьми неизвестных функций - шести компонент поля скорости, фонового давления $P_{0}$ и пространственного градиента $P_{1}$. Рассмотрим ее более подробно.

Уравнение $\dot{w}_{2}=-P_{2}^{\prime}+F_{2}^{z}$ подсистемы (20) можно переписать в силу первого соотношения подсистемы (21):

$$
\dot{w}_{2}=-P_{2}^{\prime}+F_{2}^{z}=F_{2}^{z}-\left(F_{0}^{y}\right)^{\prime}
$$

Получившееся уравнение является линейным неоднородным обыкновенным дифференциальным уравнением с постоянными коэффициентами относительно пространственного ускорения $w_{2}$ (при известном распределении массовых сил). Его решение легко находится:

$$
w_{2}=\int\left(F_{2}^{z}-\left(F_{0}^{y}\right)^{\prime}\right) d t
$$

поэтому всюду далее можно считать компоненту $w_{2}$ известной функцией.

Уравнения $\dot{u}_{1}=-P_{11}+v u_{1}^{\prime \prime}+F_{1}^{x}, \dot{u}_{2}=-P_{12}+v u_{2}^{\prime \prime}+F_{2}^{x}$ системы (20) являются нестационарными уравнениями типа неоднородного одномерного уравнения теплопроводности, решение которого находится методом декомпозиции. Интегрирование уравнений такого типа производится с помощью стандартных методик, поэтому сам алгоритм интегрирования здесь не приводится, и всюду далее мы считаем пространственные ускорения $u_{1}, u_{2}$ известными функциями. Зная вид пространственного ускорения $u_{1}$, определяем вид однородной компоненты вертикальной скорости:

$$
W=-\int u_{1} d z
$$


Затем это выражение подставляем в уравнение $\dot{W}=-P_{0}^{\prime}+v W^{\prime \prime}+F_{0}^{z}$ и определяем фоновое давление $P_{0}$ :

$$
P_{0}=v W^{\prime}+\int\left(F_{0}^{z}-\dot{W}\right) d z
$$

Таким образом, остались два уравнения $\left(\dot{U}=-P_{1}+v U^{\prime \prime}+F_{0}^{x}\right.$ и $\left.\dot{w}_{1}=-P_{1}^{\prime}+F_{1}^{z}\right)$ подсистемы (20), связывающие три неизвестных функции - продольный градиент давления $P_{1}$, скорость $U$ и пространственное ускорение $w_{1}$. Заметим, что сложившаяся ситуация обосновывается недоопределенностью системы. Таким образом, для получения решения этих двух уравнений необходимо знание дополнительных фактов о структуре хотя бы одной из трех указанных функций, например, о продольном градиенте $P_{1}$ поля давления $P$.

\section{7. Заключение}

В статье приведен новый класс точных решений уравнений Навье-Стокса, описывающий изотермические течения вязких жидкостей в поле массовых сил. Отличительной особенностью указанных решений является трехмерное представление для вертикальной компоненты вектора скорости, а также кубическое представление для поля давления и поля объемных сил. Получена редуцированная система дифференциальных уравнений как для случая установившихся, так и неустановившихся течений. Обсуждены некоторые вопросы совместности получаемых соотношений. Показано, что данные системы допускают аналитическое интегрирование, при том однозначным образом.

\section{Литература}

1. Шабловский О. Н. Сферическое течение идеальной жидкости в пространственнонеоднородном силовом поле // Вестн Том. гос. ун-та. Математика и механика. - 2020. - № 64. C. 146-155. - DOI: 10.17223/19988621/64/11.

2. Мануйлович С. В. Продольно-периодическое течение вязкой жидкости, порождаемые пристеночной объемной силой // Известия РАН. Механика жидкости и газа. - 2015. - № 4. C. 59-67.

3. Мануйлович С. В. Управление поперечным течением в пограничном слое на скользящем крыле с помощью пристеночной объемной силы // Известия РАН. Механика жидкости и газа. - 2020. - № 1. - C. 45-56. - DOI: 10.31857/S0568528120010090.

4. Rosensweig R. E. Magnetic fluids // Ann. Rev. Fluid Mech. - 1987. - Vol. 19, no. 1. P. 437-463. - DOI: 10.1146/annurev.fl.19.010187.002253.

5. $\quad$ Розенцвейг Р. Феррогидродинамика / пер. с англ. - М. : Мир, 1989. - 352 с.

6. Yerin C. V., Vivchar V. I. Ellipsometry of magnetic fluid in a magnetic field // Journal of Magnetism and Magnetic Materials. - 2020. - Vol. 498. - P. 166144. - DOI: 10.1016/j.jmmm.2019.166144.

7. Wei Pan, Jianghong Gong. Effects of magnetic fluid on magnetic fluid damper // Key Engineering Materials. - 2012. - Vols. 512-515. - P. 1479-1483. - DOI: 10.4028/www.scientific.net/KEM.512515.1479 .

8. Modification of diamagneticmaterials using magnetic fluids / I. Safarik, J. Prochazkova, E. Baldikova, M. Timko, P. Kopcansky, M. Rajnak, N. Torma, K. Pospiskova // Ukr. J. Phys. - 2020. Vol. 65, no. 9. - P. 751-760. - DOI: 10.15407/ujpe65.9.751.

9. Horváth B., Szalai I. Nonlinear magnetic properties of magnetic fluids for automotive applications // Hungarian Journal of Industry and Chemistry. - 2020. - Vol. 48, no. 1. - P. 61-65. DOI: $10.33927 /$ hjic-2020-09. 
10. Брацун Д. А., Вяткин В. А., Мухаматуллин А. Р. О точных нестационарных решениях уравнений вибрационной конвекции // Вычислительная механика сплошных сред. - 2017. T. 10, № 4. - C. 433-444. - DOI: 10.7242/1999-6691/2017.10.4.35.

11. Конвективные процессы в невесомости / В. И. Полежаев, М. С. Белло, Н. А. Верезуб, К. Г. Дубовик, А. П. Лебедев, С. А. Никитин, Д. С. Павловский, А. И. Федюшкин. - М. : Наука, 1991. - 242 с.

12. Полежаев В. И., Сазонов В. В. Механика невесомости и гравитационночувствительные системы. Аннотации докладов научно-исследовательского семинара. М. : Институт проблем механики РАН им. В. М. Келдыша (препринт № 898), 2009. - 76 с.

13. Суржиков С. Т. Гиперзвуковое обтекание разреженным газом поверхностного тлеющего разряда с внешним магнитным полем. - М. : Ин-т проблем механики РАН, 2011. $274 \mathrm{c}$.

14. Kedrinskiy V. K. Role of nuclei density as a "hidden" parameter in the formation of anomalous zones in a heavy cavitating magma // Journal of Applied Mechanics and Technical Physics. 2014. - Vol. 55. - P. 276-281. - DOI: 10.1134/S0021894414020102.

15. Afonin V. V., Petrov V. Y. On exact solutions for a Luttinger liquid with one impurity // Jetp Lett. - 2019. - Vol. 109. - P. 762-767. - DOI: 10.1134/S0021364019110031.

16. Kouwn S., Moon T., Oh P. Exact solution and exotic fluid in cosmology // Entropy. - 2012. Vol. 14. - P. 1771-1783. - DOI: 10.3390/e14091771.

17. Аристов С. Н. Стационарный цилиндрический вихрь в вязкой жидкости // Доклады Академии наук. - 2001. - Т. 377. - С. 477-480.

18. Аристов С. Н. Точное решение задачи о точечном источнике // Доклады Академии наук. - 1995. - Т. 343, № 1. - С. 50-52.

19. Aristov S. N. Three-Dimensional Conical Viscous Incompressible Fluid Flows // Fluid Dynamics. - 1998. - Vol. 33, no. 929. - DOI: 10.1007/BF02698663.

20. Aristov S. N., Grabovskii V. I. Self-similar solution of the Navier-Stokes equations governing gas flows in rotary logarithmically spiral two-dimensional channels // Fluid Dyn. - 1995 Vol. 30. - P. 838-844. - DOI: 10.1007/BF02078198.

21. Темам Р. Уравнения Навье-Стокса. Теория и численный анализ / пер. с англ. М. : Мир, 1981. -408 с.

22. Ландау Л. Д., Лифшиц Е. М. Гидродинамика. Теоретическая физика : том VI. - Изд. 4-е, стереотипное. - М. : Наука, 1988. - 736 с.

23. Хмельник С. И. Уравнения Навье-Стокса. Существование и метод поиска глобального решения. - Израиль, Mathematics in Computer Comp., 2010. - 106 с.

24. Рауз Х. Механика жидкости. - М. : Изд. литературы по строительству, 1967. - 392 с.

25. Boussinesq J. Théorie de l'écoulement tourbillonnant et tumultueux des liquides dans les lits rectilignes a grande section. - Paris, Gauthier-Villars et fils, 1897. -90 p.

26. Гершуни Г. 3., Жуховицкий Е. М. Конвективная устойчивость несжимаемой жидкости. - М. : Наука, 1972. - 392 с.

27. Müller P. Boussinesq approximation. In the equations of oceanic motions. - Cambridge : Cambridge University Press, 2006. - DOI:10.1017/CBO9780511617843.012.

28. Бурмашева Н. В., Просвиряков Е. Ю. Точное решение для установившихся конвективных концентрационных течений типа Куэтта // Вычислительная механика сплошных сред. - 2020. - Т. 13, № 3. - C. 337-349. - DOI: 10.7242/1999-6691/2020.13.3.27.

29. Burmasheva N. V., Prosviryakov E. Yu. On Marangoni shear convective flows of inhomogeneous viscous incompressible fluids in view of the Soret effect // Journal of King Saud University Science. - 2020. - Vol. 32, no. 8. - P. 3364-3371. - DOI: 10.1016/j.jksus.2020.09.023.

30. Martyushev S. G., Sheremet M. A. Conjugate natural convection combined with surface thermal radiationin a three-dimensional enclosure with a heat source // International Journal of Heat and Mass Transfer. - 2014. - Vol. 73. - P. 340-353. - DOI: 10.1016/j.ijheatmasstransfer.2014.02.009. 
31. Couette M. Etudes sur le frottement des liquides // Ann. Chim. Phys.. - 1890. - Vol. 21. P. 433-510.

32. Poiseuille J. Recherches experimentelles sur le mouvement des liquides dans les tubes de tres petits diametres // Comptes Rendus. - 1840. - Vol. 11. - P. 961-967.

33. Stokes G. G. On the effct of the internal friction of fluid on the motion of pendulums / G.G. Stokes // Camb. Philo. Trans. - 1851. - Vol. 9. - P. 8-106.

34. Lin C. C. Note on a class of exact solutions in magneto-hydrodynamics // Arch. Rational Mech. Anal. - 1958. - Vol. 1. - P. 391-395.

35. Шмыглевский Ю. Д. Аналитические исследования динамики газа и жидкости. М. : Эдиториал УРСС, 1999. - 231 с.

36. Сидоров А. Ф. О двух классах решений уравнений механики жидкости и газа и их связи с теорией бегущих волн // ПМТФ. - 1989. - Вып. 2. - С. 34-40.

37. Аристов С. Н. Вихревые течения в тонких слоях жидкости : дис. ... докт. физ.-мат. наук : 01.02.05. - Владивосток : ИАПУ, 1990. - 330 с.

38. Аристов С. Н., Просвиряков Е. Ю. Новый класс точных решений трехмерных уравнений термодиффузии // Теоретические основы химической технологии. -2016 . - Т. 50, № 3. C. 294-301. - DOI: 10.7868/S0040357116030027.

39. Prosviryakov E. Yu. A sufficient condition for the absence of strong and weak discontinuities in gas flows in flat channels // Diagnostics, Resource and Mechanics of materials and structures. - 2019. - Iss. 3. - P. 25-40. - DOI: 10.17804/2410-9908.2019.3.025-040.

40. Аристов С. Н., Просвиряков Е. Ю. Крупномасштабные течения завихренной вязкой несжимаемой жидкости // Известия высших учебных заведений. Авиационная техника. 2015. - Вып. 4. - C. 50-54.

41. Privalova V.V., Prosviryakov E.Yu. Vortex flows of a viscous incompressible fluid at constant vertical velocity under perfect slip conditions // Diagnostics, Resource and Mechanics of materials and structures. - 2019. - Вып. 2. - C. 57-70. - DOI: 10.17804/2410-9908.2019.2.057-070.

42. Privalova V. V., Prosviryakov E. Yu. Exact solutions for the convective creep CouetteHiemenz flow with the linear temperature distribution on the upper border // Diagnostics, Resource and Mechanics of materials and structures. - 2018. - Iss. 2. - P. 92-109. - DOI: 10.17804/24109908.2018.2.092-109.

43. Privalova V. V. and Prosviryakov E. Yu. The effect of tangential boundary stresses on the convective unidirectional flow of a viscous fluid layer under the lower boundary heating condition // Diagnostics, Resource and Mechanics of materials and structures. - 2019. - Iss. 4. - P. 44-55. DOI: $10.17804 / 2410-9908.2019 .4 .044-055$.

44. Burmasheva N. V., Prosviryakov E. Yu. An exact solution to the description of a unidirectional Marangoni flow of a viscous incompressible fluid with the Navier boundary condition. Velocity field investigation // Diagnostics, Resource and Mechanics of materials and structures. 2019. - Iss. 5. - P. 23-39. - DOI: 10.17804/2410-9908.2019.5.023-039.

45. Burmasheva N. V., Prosviryakov E. Yu. An exact solution for describing the unidirectional Marangoni flow of a viscous incompressible fluid with the Navier boundary condition. Temperature field investigation // Diagnostics, Resource and Mechanics of materials and structures. - 2020. Iss. 1. - P. 6-23. - DOI: 10.17804/2410-9908.2020.1.006-023.

46. Burmasheva N. V., Prosviryakov E. Yu. Exact solution for describing a unidirectional Marangoni flow of a viscous incompressible fluid with the Navier boundary condition. Pressure field investigation // Diagnostics, Resource and Mechanics of materials and structures. - 2020. - Iss. 2. P. 61-75. - DOI: 10.17804/2410-9908.2020.2.061-075.

47. Burmasheva N.V., Prosviryakov E.Yu. Temperature field investigation in layered flows of a vertically swirling viscous incompressible fluid under two thermocapillar forces at a free boundary // Diagnostics, Resource and Mechanics of materials and structures. - 2019. - Iss. 1. - P. 6-42. DOI: 10.17804/2410-9908.2019.1.006-042. 
48. Gorshkov A.V., Prosviryakov E.Y. Complex stationary convection with third-kind boundary conditions at the boundaries of a fluid layer // Diagnostics, Resource and Mechanics of materials and structures. - 2016. - Iss. 2. - P. 34-47. - DOI: 10.17804/2410-9908.2016.2.034-047.

49. Burmasheva N.V., Prosviryakov E.Yu. Exact solutions for natural convection of layered flows of a viscous incompressible fluid with Specified tangential forces and the linear distribution of temperature on the layer boundaries // Diagnostics, Resource and Mechanics of materials and structures. - 2017. - Iss. 4. - P. 16-31. - DOI: 10.17804/2410-9908.2017.4.016-031.

50. Petrov A. G. Exact solution of the Navier-Stokes equations in a fluid layer between the moving parallel plates // Journal of Applied Mechanics and Technical Physics. - 2012 - Vol. 53, no. 5. P. 642-646. - DOI: 10.1134/S0021894412050021.

51. Зырянов В. Н. Теория установившихся океанических течений. - Ленинград : Гидрометеоиздат, 1985.

52. Бурмашева Н. В., Просвиряков Е. Ю. Точное решение уравнений Навье-Стокса, описывающее пространственно неоднородные течения вращающейся жидкости // Труды Института математики и механики УрО РАН. - 2020. - Т. 26, вып. 2. - С. 79-87. DOI: $10.21538 / 0134-4889-2020-26-2-79-87$.

53. Бурмашева Н. В., Просвиряков Е. Ю. Класс точных решений для двумерных уравнений геофизической гидродинамики с двумя параметрами Кориолиса // Известия Иркутского государственного университета. Серия Математика. - 2020. - Т. 32. - С. 33-48. DOI: $10.26516 / 1997-7670.2020 .32 .33$.

54. Burmasheva N. V., Prosviryakov E. Yu. Isothermal layered flows of a viscous incompressible fluid with spatial acceleration in the case of three Coriolis parameters // Diagnostics, Resource and Mechanics of materials and structures. - 2020. - Iss. 3. - P. 29-46. - DOI: 10.17804/24109908.2020.3.029-046.

55. Meleshko S. V., Pukhnachev V. V. On a class of partially invariant solutions of the NavierStokes equations // Journal of Applied Mechanics and Technical Physics. - 1999. - Vol. 40, no. 2. P. 208-216.

56. Пухначев В. В. Симметрии в уравнениях Навье-Стокса // Успехи механики. - 2006. T. 1. - C. 6-76.

57. Aristov S. N., Gitman I. M. Viscous flow between two moving parallel disk: exact solutions and stability analysis // J. Fluid Mech. - 2002. - Vol. 464. - P. 209-215. DOI: $10.1017 /$ S0022112002001003.

58. Berker R. An exact solution of the Navier-Stokes equation - the vortex with curvilinear axis // Int. J. Eng. Sci. - 1982. - Vol. 20. - P. 217-230.

59. Berker R. A new solution of the Navier-Stokes equation for the motion of a fluid contained between two parallel plates rotating about the same axis // Arch. Mech. - 1979. - Vol. 31. - P. 265-280.

60. Dorrepaal J. M. An exact solution of the Navier-Stokes equation which describes nonorthogonal stagnation point flow in two dimensions // J. Fluid Mech. - 1986. - Vol. 163. - P. 141-147.

61. Ekman V. W. On the influence of the earth's rotation on ocean currents // Ark. Mat. Astron. Fys. - 1905. - Vol. 2. - P. 1-52.

62. Gupta A. S. Ekman layer on a porous plate // Phys. F1uids. - 1972. - Vol. 15. - P. 930-931.

63. Marques F., Sanchez J., Weidman P. D. Generalized Couette - Poiseuille flow with boundary mass transfer // J. Fluid Mech. - 1998. - Vol. 374. - P. 221-249.

64. Rajagopal K. R. A class of exact solutions to the Navier-Stokes equations // Int. J. Eng. Sci. - 1984. - Vol. 22. - P. 451-458.

65. Rasmussen H. Steady flow between two porous disks // ZAMP. - 1970. - Vol. 21. - P. 187-195.

66. Wang C. Y. Exact solution of the Navier-Stokes equations-the generalized Beltrami flows, review and extension // Acta Mech. - 1990. - Vol. 81. - P. 69-74.

67. Wang C. Y. Exact solutions of the steady-state Navier-Stokes equations // Annual Review of Fluid Mechanics - 1991. - Vol. 23. - P. 159-177. 
68. Wang C. Y. Stagnation flow with slip: Exact solution of the Navier-Stokes equations // ZAMP. - 2003. - Vol. 54. - P. 184-189.

69. Wang C. Y. On a class of exact solutions of the Navier-Stokes equations // J. Appl. Mech. 1966. - Vol. 33. - P. 696-698. 